OPEN ACCESS

Edited by: Javier Ramírez,

University of Granada, Spain

Reviewed by:

Yvonne Höller

Paracelsus Private Medical University

of Salzburg, Austria

Andres Ortiz,

University of Málaga, Spain

*Correspondence:

Tong Boon Tang

tongboon.tang@utp.edu.my

${ }^{\dagger}$ These authors have contributed equally to this work.

Received: 17 May 2017 Accepted: 17 August 2017 Published: 01 September 2017

Citation:

Yap KH, Ung WC, Ebenezer EGM, Nordin N, Chin PS, Sugathan S,

Chan SC, Yip HL, Kiguchi M and

Tang TB (2017) Visualizing Hyperactivation in Neurodegeneration Based on Prefrontal Oxygenation: A Comparative Study of Mild Alzheimer's Disease, Mild Cognitive Impairment, and Healthy Controls.

Front. Aging Neurosci. 9:287. doi: 10.3389/fnagi.2017.00287

\section{Visualizing Hyperactivation in Neurodegeneration Based on Prefrontal Oxygenation: A Comparative Study of Mild Alzheimer's Disease, Mild Cognitive Impairment, and Healthy Controls}

\author{
Kah Hui Yap ${ }^{1 \dagger}$, Wei Chun Ung ${ }^{2 \dagger}$, Esther G. M. Ebenezer ${ }^{1}$, Nadira Nordin ${ }^{2}$, Pui See Chin ${ }^{1}$, \\ Sandheep Sugathan ${ }^{3}$, Sook Ching Chan ${ }^{3}$, Hung Loong Yip ${ }^{3}$, Masashi Kiguchi ${ }^{4}$ and \\ Tong Boon Tang ${ }^{2 *}$ \\ ${ }^{1}$ Medicine Based Department, Royal College of Medicine Perak, Universiti Kuala Lumpur, Kuala Lumpur, Malaysia, ${ }^{2}$ Centre \\ for Intelligent Signal and Imaging Research, Universiti Teknologi Petronas, Seri Iskandar, Malaysia, ${ }^{3}$ Community Based \\ Department, Royal College of Medicine Perak, Universiti Kuala Lumpur, Kuala Lumpur, Malaysia, ${ }^{4}$ Research \& Development \\ Group, Hitachi Ltd., Tokyo, Japan
}

Background: Cognitive performance is relatively well preserved during early cognitive impairment owing to compensatory mechanisms.

Methods: We explored functional near-infrared spectroscopy (fNIRS) alongside a semantic verbal fluency task (SVFT) to investigate any compensation exhibited by the prefrontal cortex (PFC) in Mild Cognitive Impairment (MCl) and mild Alzheimer's disease (AD). In addition, a group of healthy controls $(\mathrm{HC})$ was studied. A total of 61 volunteers (31 $\mathrm{HC}, 12$ patients with $\mathrm{MCl}$ and 18 patients with mild $\mathrm{AD}$ ) took part in the present study.

Results: Although not statistically significant, $\mathrm{MCl}$ exhibited a greater mean activation of both the right and left PFC, followed by $\mathrm{HC}$ and mild $\mathrm{AD}$. Analysis showed that in the left PFC, the time taken for $\mathrm{HC}$ to achieve the activation level was shorter than $\mathrm{MCl}$ and mild $\mathrm{AD}(p=0.0047$ and 0.0498 , respectively); in the right $\mathrm{PFC}$, mild $\mathrm{AD}$ took a longer time to achieve the activation level than $\mathrm{HC}$ and $\mathrm{MCl}(p=0.0469$ and 0.0335 , respectively); in the right PFC, $\mathrm{HC}$, and $\mathrm{MCl}$ demonstrated a steeper slope compared to mild $\mathrm{AD}$ $(p=0.0432$ and 0.0107 , respectively). The results were, however, not significant when corrected by the Bonferroni-Holm method. There was also found to be a moderately positive correlation $(R=0.5886)$ between the oxygenation levels in the left PFC and a clinical measure [Mini-Mental State Examination (MMSE) score] in $\mathrm{MCl}$ subjects uniquely.

Discussion: The hyperactivation in $\mathrm{MCl}$ coupled with a better SVFT performance may suggest neural compensation, although it is not known to what degree hyperactivation manifests as a potential indicator of compensatory mechanisms. However, hypoactivation plus a poorer SVFT performance in mild AD might indicate an inability to compensate due to the degree of structural impairment. 
Conclusion: Consistent with the scaffolding theory of aging and cognition, the task-elicited hyperactivation in $\mathrm{MCl}$ might reflect the presence of compensatory mechanisms and hypoactivation in mild $A D$ could reflect an inability to compensate. Future studies will investigate the fNIRS parameters with a larger sample size, and their validity as prognostic biomarkers of neurodegeneration.

Keywords: mild Alzheimer's disease, mild cognitive impairment, functional near-infrared spectroscopy, semantic verbal fluency task, prefrontal hemoglobin oxygenation

\section{INTRODUCTION}

The multiple dementia subtypes are associated with unique symptom patterns and brain abnormalities. Alzheimer's disease $(\mathrm{AD})$ is a chronic progressive neurodegenerative brain disease that can occur in middle or old age. It represents the most common cause of dementia, accounting for $60-80 \%$ of cases (Alzheimer's Association, 2016). It causes increasing impairment in a range of cognitive functions that include memory, mood, reasoning, language, self-management, and behavior (Karantzoulis and Galvin, 2014). Mild cognitive impairment (MCI) is an intermediate state of clinical impairment, where the individuals affected have cognitive symptoms of a mild nature that are disproportionate to their age and education, while not meeting the criteria for dementia or AD (Petersen, 2009). Patients with $\mathrm{MCI}$ tend to progress toward developing $\mathrm{AD}$ at a rate of $\sim 15 \%$ per year (Gauthier et al., 2006).

Magnetic resonance imaging (MRI) studies have demonstrated that deficits found in patients with $\mathrm{AD}$ are associated with volumetric changes in the prefrontal cortex (PFC) (Salat et al., 2001; McNab and Klingberg, 2008; Zanto et al., 2011). It has been suggested that the cognitive decline related to normal aging is attributable to a reduction in white matter rather than gray matter (Marner et al., 2003). In contrast, significant loss of gray matter, which is composed of cortical neurons and glia has been seen in $\mathrm{AD}$, and leads to a reduced neuronal activation in the $\mathrm{PFC}$ of $\mathrm{AD}$ when compared with HC (Yankner et al., 2008). MCI is positioned between mild AD and normal cognitive aging (Sperling, 2007). While age-related regional volume loss is apparent and widespread in normal cognitive aging, a unique pattern of structural vulnerability, reflected in differential volume loss in specific regions, has been identified in patients with MCI (Driscoll et al., 2009). Also, patients with $\mathrm{AD}$ perform more poorly in semantic tasks associated with compromised activation in the left PFC when assessed using functional MRI (fMRI) (Johnson et al., 2000). The

\footnotetext{
Abbreviations: AD, Alzheimer's disease; aMCI, amnestic mild cognitive impairment; $A \beta$, amyloid $\beta$-protein; $C D R$, clinical dementia rating; deoxy$\mathrm{Hb}$, deoxygenated-hemoglobin; fMRI, functional magnetic resonance imaging; fNIRS, functional near-infrared spectroscopy; HC, healthy controls; MCI, Mild cognitive impairment; MMSE, mini-mental state examination; MRI, magnetic resonance imaging; naMCI, Non-amnesic mild cognitive impairment; oxy$\mathrm{Hb}$, oxygenated-hemoglobin; PFC, prefrontal cortex; SVFT, semantic verbal

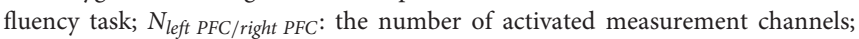

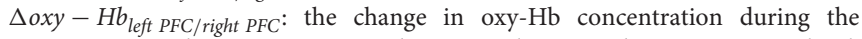

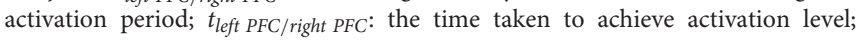

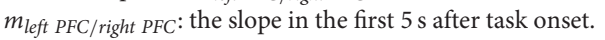

hippocampus is among the first non-cortical regions affected by $\mathrm{AD}$-related neurodegeneration; hippocampal atrophy has been associated with early memory decline in MCI and AD (Barnes et al., 2007; Shi et al., 2009; Nho et al., 2012). Several previous studies of MCI patients and healthy older adults reported reduced activation in the hippocampus and PFC of the former (Johnson et al., 2006; Dannhauser et al., 2008; Mandzia et al., 2009). These results suggest that AD may be characterized by reduced brain activation due to the pathological changes associated with it.

One key element influencing these early changes is neural compensation. The capacity for neural compensation is inversely proportional to the severity of neurodegeneration (Price and Friston, 2002). As neural damage worsens, both cognitive processing efficiency and capacity become impeded. This affects neuronal recruitment capacity, reduces compensation capacities, and ultimately results in poorer performance (Scarmeas et al., 2003). As an example, transcranial magnetic stimulation has been shown to induce compensatory activation associated with information retrieval when applied to either the right or left PFC of patients with severe cognitive impairment. However, similar activation was only observed when applied to the left PFC of healthy individuals. These data suggest that right PFC recruitment acts as one of the functional compensatory mechanisms in cognitively impaired individuals (Cotelli et al., 2008). In addition, neural compensation prolonged the period of MCI and delayed progression to AD (Baazaoui et al., 2017), implying that such compensatory mechanisms may play a more crucial role in MCI than in $\mathrm{AD}$. In the present study, the aim was to investigate the difference between MCI and $\mathrm{AD}$ using functional near-infrared spectroscopy (fNIRS) to identify hyperactivation as a potential indicator of the occurrence of compensation.

fNIRS is an emerging technology in neuroimaging that has been increasingly employed in the past 20 years (Ferrari and Quaresima, 2012). It uses an optical window where the head scalp is almost transparent to near infrared light at wavelengths of 700-900 nm, and the fact that oxygenated- and deoxygenatedhemoglobin (oxy-Hb and deoxy-Hb) are strong absorbers of light (Heinzel et al., 2013). Based on the concept of neurovascular coupling, neuronal activities are measured by fNIRS as changes in oxy-Hb and deoxy-Hb concentrations using the Modified Beer-Lambert Law. fNIRS has, so far, been applied to many psychiatric disorders, for example, to differentiate depression, bipolar disorder, and schizophrenia (Fallgatter et al., 1997; Arai et al., 2006; Takizawa et al., 2014). The technique has a good 
temporal resolution $(\sim 1 \mathrm{~ms})$ and a reasonable spatial resolution $(\sim 1 \mathrm{~cm})$. In addition, it has several advantages over the more widely-used fMRI, including lower costs, portability, and low levels of subject constraint (Ehlis et al., 2014). It has been demonstrated that fNIRS readings are highly correlated with fMRI with respect to measuring cognitive tasks (Cui et al., 2011). More specifically, multiple fNIRS-fMRI studies have consistently shown increases in oxy-Hb in the left PFC during semantic verbal fluency tasks (SVFT) (Fallgatter et al., 1997; Heinzel et al., 2013; Wagner et al., 2014; Gutierrez-Sigut et al., 2015). So, fNIRS represents a potential alternative technique to fMRI for the investigation of the differences in neuronal activities between individuals with differing cognitive impairments.

fNIRS has been used in previous studies into AD and MCI. Patients with $\mathrm{AD}$ were identified to have lower activation of the PFC compared to healthy controls (HC) during both letter and SVFT, while HC performed better in the tasks. Differences in hemodynamic responses were predominantly found in the left hemisphere, supporting the idea that good performances in verbal fluency tasks are associated with higher left hemisphere activation, predominantly (Fallgatter et al., 1997; Arai et al., 2006; Herrmann et al., 2008). This poorer performance in patients with $\mathrm{AD}$ has been attributed to the loss of hemispheric asymmetry rather than to the level of PFC activation alone (Fallgatter et al., 1997). The effect of hemispheric asymmetry or lateralization plays a more crucial role in MCI. The rightward shift of frontal activations in the MCI group might reflect the presence of cortical reorganization; the recruitment of the right $\mathrm{PFC}$ has been suggested to compensate the loss in the left PFC (Yeung et al., 2016). Another study has shown that compared to HC, patients with $\mathrm{AD}$ demonstrated lower activation of the frontal and bilateral parietal areas, while patients with MCI have lower right parietal activation (Arai et al., 2006). These results suggest that more detailed studies into neural compensatory mechanisms are warranted.

In the present study, fNIRS was used with a wide coverage of the PFC to investigate hyper/ hypoactivation in MCI, mild $\mathrm{AD}$, and $\mathrm{HC}$. The PFC was selected as the region of interest as the PFC is not only involved in semantic memory, but is also accessible by fNIRS, which can penetrate brain tissue up to a depth of $5 \mathrm{~cm}$ in the cortical region (Ranger et al., 2011). SVFT was selected as the cognitive activation task based on previous studies of semantic memory (Fallgatter et al., 1997; Thompson-Schill et al., 1997; Perry et al., 2000; Grossman et al., 2002; Heinzel et al., 2013; Wagner et al., 2014; GutierrezSigut et al., 2015; Yeung et al., 2016). In accordance with the compensation theory (Yankner et al., 2008), it was expected that neural compensation would be observed in MCI, but not in $\mathrm{AD}$ during the tasks. Patients with MCI suffer from a relatively small degree of neurodegeneration compared to patients with $\mathrm{AD}$, therefore, it was predicted that MCI patients would be able to activate neural compensation, as manifested by brain hyperactivation, in order to maintain task performance. As patients with $\mathrm{AD}$ suffer from more severe neuronal damage, to the extent that their neural compensation abilities might be compromised, it was thought that hypoactivation would be observed instead. We aimed to investigate to what degree hyperactivation, as a potential indicator for compensation, manifests in MCI and to what degree it is compromised in AD. Our first hypothesis was that during SVFT, subjects with normal cognitive aging (the HC group) would perform better, followed by MCI and AD. Secondly, we expected hyperactivation and hypoactivation in the PFC of MCI and AD, respectively, when subjects were tested with a semantic memory task.

\section{METHODS}

\section{Participants}

We recruited participants, who were right-handed and able to converse in English, through a local dementia day-care center as well as from the local community where English was the common medium of instruction in the past. Patients with MCI and patients with mild AD were recruited through purposive sampling with group-specific inclusion criteria. This was followed by the recruitment of $\mathrm{HC}$ who were age ( \pm 2 years), gender- and education-matched. We assessed the participants, ruling out anyone with a psychiatric disorder. Additionally, neurological disorders, including other forms of dementia were excluded. Assessments were performed by a psychiatrist using an evaluation of medical history, including careful examination of the course of progression, the relative salience of cognitive, behavioral and physical symptoms and signs, and patterns of cognitive impairment. Additionally, mental state was examined using a Mini-Mental State Examination (MMSE), which is a 30-point questionnaire providing a quantitative measure of cognitive status or cognitive impairment (Folstein et al., 1975). Other exclusion criteria were other medical diagnoses affecting cognitive functioning, including kidney failure, stroke, known lesions, and any history of significant trauma. The study protocol was approved by the Medical Research Ethics Committee of the University of Kuala Lumpur (Approval no.: 2015/032). All participants were briefed about the nature of the experimental procedures prior to providing demographic information and written informed consent in accordance with the Declaration of Helsinki. All tests and experiments were completed on the same day, with a short break between the test and the experiment. Both healthy participants and patients were remunerated for their participation.

\section{Clinical Measures}

We used the Clinical Dementia Rating (CDR), an observer rating scale designed to rate the severity of dementia (Morris, 1993), for the diagnosis of dementia and group allocation. CDR scores of 0 , 0.5 , and 1 were assigned to $\mathrm{HC}, \mathrm{MCI}$, and mild $\mathrm{AD}$, respectively; participants with CDR scores of 2 (moderate) and 3 (severe) were excluded, as this study focused on MCI and mild AD. In addition, we assessed and assigned each participant an MMSE score. CDR has a moderate to high inter-rater reliability of 0.62 (Rockwood et al., 2000). MMSE has high inter-rater reliability, ranging between 0.82 and 0.91 (Magni et al., 1996). We matched the $\mathrm{HC}$ to those of the combined sample of patients according to age, sex, and education. 


\section{fNIRS Technology}

Throughout this study, a multichannel OT-R40 fNIRS topography system (Hitachi Medical Corporation, Japan) was employed to measure the brain activity at a sampling rate of $10 \mathrm{~Hz}$. Changes in oxy- $\mathrm{Hb}$ and deoxy-Hb signals were measured in units of $\mathrm{mM} \cdot \mathrm{mm}$. ANIRS has been reported to be more sensitive to gray matter when a larger source-detector separation (up to $\sim 4.5 \mathrm{~cm}$ ) is used, albeit at the expense of both spatial resolution and partial pathlength factors (Strangman et al., 2013). Taking everything into consideration, the sourcedetector distance was fixed at $3 \mathrm{~cm}$, within the suggested optimal range for adult heads $(3-3.5 \mathrm{~cm}$ ) (Li et al., 2011). The midpoint between pairs of sources and the detector was defined as a measurement channel. The probes were arranged into a $3 \times 11$ layout (see Figure 1A) to form a total of 52 measurement channels that were sufficient to measure the entire PFC and part of the temporal cortex (see Figure 1B; Ishii-Takahashi et al., 2014; Takizawa et al., 2014). According to the international 10-20 system (Klem et al., 1999), source no. 23 and 28 were positioned directly at T4 and T3, respectively. The probes were attached to a flexible head cap, which was relatively easy, fast and convenient to wear. All channels were checked to ensure that the probes were in contact with the scalp. The entire set-up process took an average of $<10 \mathrm{~min}$.

\section{Task Paradigm}

Participants were seated comfortably in a working chair and were instructed to avoid movement and to place their hands on the armrests during the experiment. Standardized verbal instructions and explanations regarding the tasks were given in English. Prior to any new measurement, practice was given, allowing the participants to familiarize themselves with the experimental procedures. SVFT was selected as the cognitive activation task in this study. During fNIRS measurements, participants were instructed to provide as many words verbally as possible from a particular category (Fruits, Food, and Animals). The experimental session was preceded by $20 \mathrm{~s}$ of pre-task rest period. Each category lasted $60 \mathrm{~s}$ and was followed by $20 \mathrm{~s}$ of rest. Participants were told to avoid repeating the same word and they were asked to keep their eyes on the LCD screen for the entire task period, which lasted for a total of $260 \mathrm{~s}$ (see Figure 2).

\section{Data Analysis fNIRS Data}

Oxy-Hb was selected as the focus of measurements due to its sensitivity to task-associated changes (Sato et al., 2006; Cui, 2011). Probes that were not in good contact with the scalp may have resulted in rapid large changes (such as high amplitude spikes) in oxy-Hb signals. An fNIRS channel was considered to be "noisy" if there were very large spikes (changes in oxy- $\mathrm{Hb}$

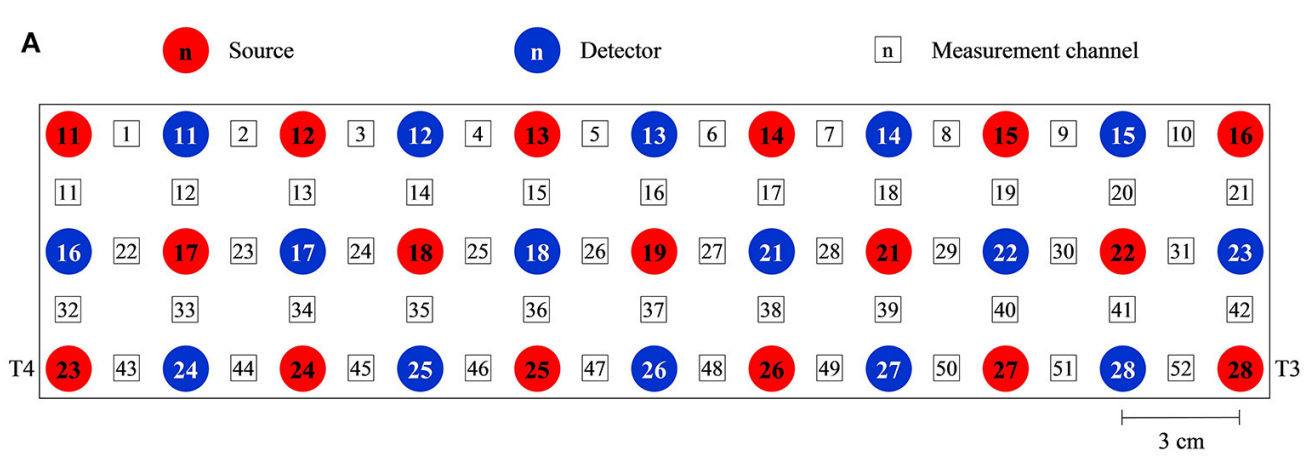

8
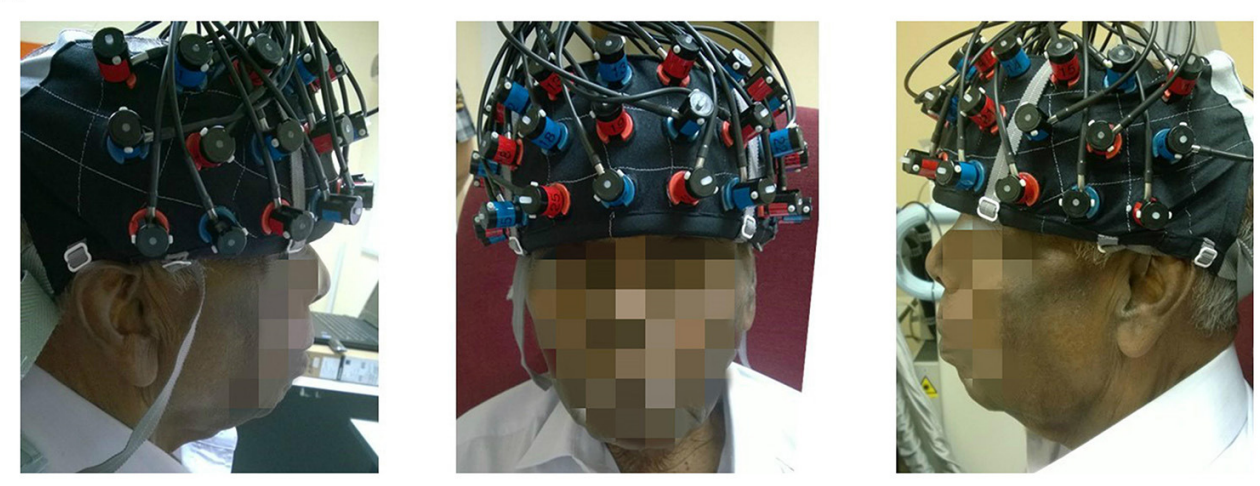

FIGURE 1 | A system consisting of 52 measurement channels was used. (A) The probes were arranged to $3 \times 11$ layout. The source-detector distance was fixed at $3 \mathrm{~cm}$ and the space between pairs of source and detector was defined as a measurement channel. (B) One of the participants wearing the flexible head cap which housed the probes. Source no. 23 and 28 were positioned directly at T4 and T3 accordingly to the international 10-20 system. Consent was obtained from the individual for the publication of this image. 


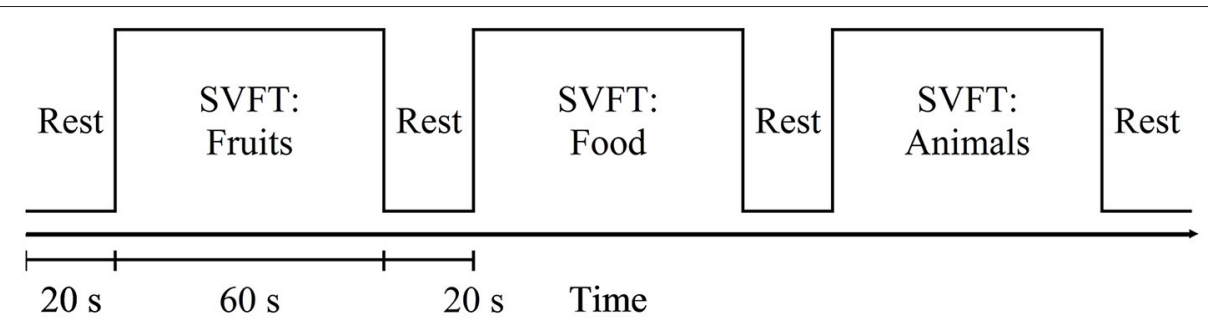

FIGURE 2 | Schematic diagram of the measurement sequence: SVFT = semantic verbal fluency test. It started with a 20 s rest. Each category of SVFT was 60 s long and was followed by 20 s of rest.

larger than $0.5 \mathrm{mM} \cdot \mathrm{mm}$ in amplitude) and all noisy channels were excluded from subsequent analyses. All data analyses were performed using a software Platform for Optical Topography Analysis Tools (Sutoko et al., 2016). A Butterworth bandpass filter with cut-off frequencies of $0.01-0.8 \mathrm{~Hz}$ was applied to remove instrumental or physiological noise (Luu and Chau, 2009).

Three categories of SVFT were represented by three 100-s blocks of data for analysis. As discussed in the task paradigm, each block consisted of a 20-s pre-task rest period, a 60-s task period followed by a 20-s post-task period (see to Figure 3). A moving average filter with a window size of 50 data points ( $5 \mathrm{~s}$ ) was applied to remove high frequency noise from the measured oxy-Hb signals (Ishii-Takahashi et al., 2014). With respect to the start of each block, the oxy-Hb signals were then baselinecorrected to a zero baseline. Blocks with a large spike noise in the oxy-Hb signal (changes in oxy-Hb larger than $0.5 \mathrm{mM} \cdot \mathrm{mm}$ in amplitude) were excluded from further analyses. Subsequently, fNIRS signals from each channel were meaned over all the remaining blocks so that each subject should have had only one average fNIRS signal per channel. Subsequent analyses were performed using these average fNIRS signals. Previous fNIRS studies have suggested that earliest activation starts from $5 \mathrm{~s}$ after task onset (Sato et al., 2006) and sharp increases in activation are often observed at around 5-10 s after onset (Maki et al., 1995). Therefore, the level of activation at each channel was determined for each individual using the percentage signal change, which was calculated using the following formula:

$$
\begin{aligned}
& \text { percent signal change } \\
& \qquad=\frac{\text { oxy }-H b_{t=5: 65(\mathrm{avg})}-o x y-H b_{t}=-10: 0(\mathrm{avg})}{\mid \text { oxy }-H b_{t}=-10: 0(\mathrm{avg}) \mid} \times 100 \%
\end{aligned}
$$

where $o x y-H b_{t}=5: 65$ (avg) is the average oxy-Hb signal during the task period, after accounting for hemodynamic delay, and $o x y-H b_{t}=-10: 0$ (avg) is the average oxy-Hb signal during the rest period $(-10-0 \mathrm{~s}$ of the pre-task rest period; see Figure 3). Channels located in the PFC that showed a percentage signal change of larger than $50 \%$ were empirically considered to be activated and were further divided into left and right PFC. Hence, it was possible for each subject to have a different number of activated channels, but they were all within the regions of interest (the left and right PFC).

Due to hemodynamic delay, the period between 5 and $25 \mathrm{~s}$ after task onset was defined as the activation period (see Figure 3). The activation signal was defined as the difference between the average oxy- $\mathrm{Hb}$ signal during the activation period and during the rest period. For each participant, the mean activation signal in the left and right PFC was calculated by averaging the signals obtained in activated measurement channels located in the left and right PFC, respectively. Based on the mean activation signals, for both the left and right PFC, the time taken to achieve the activation level was determined and the slope in the first $5 \mathrm{~s}$ after task onset was calculated using the following formula:

$$
\begin{aligned}
\text { slope }= & \frac{o x y-H b_{\text {left } P F C / \text { right } P F C}^{t=0 x y}-H b_{\text {left } P F C / \text { right } P F C}^{t=0}}{5} \\
& \times \frac{\mathrm{mM} \cdot \mathrm{mm}}{\mathrm{s}}
\end{aligned}
$$

where oxy $-H b_{\text {left } P F C / \text { right } P F C}^{t=0}$ and $o x y-H b_{\text {left PFC/right } P F C}^{t=5}$ denote the mean activation signals in the left or right PFC at the onset of the task and $5 \mathrm{~s}$ after the onset of the task, respectively.

The number of activated measurement channels, the mean activation signals in both the left and right PFC, and the time taken to achieve the activation level and the slope in the first $5 \mathrm{~s}$ after task onset were then statistically assessed.

\section{Statistics}

Differences between all three groups in MMSE scores were first tested using the multiple non-parametric Mann-Whitney U tests without multiple testing correction, followed by MannWhitney U tests with Bonferroni-Holm correction. Both tests were repeated to assess whether there were any within-category group differences in the number of words generated in the three categories of SVFT. The Bonferroni-Holm correction would test each individual hypothesis in a sequential rejective manner at $\alpha /[n-$ rank number of the pair (by degree of significance) +1$]$, where $\alpha$ is the desired significance level (0.05) and $n$ is the number of comparisons. fNIRS data that were statistically assessed included the number of activated measurement channels, the mean activation signals in both the left and right PFC, the time taken to achieve the activation level and the slope in the first $5 \mathrm{~s}$ after task onset. For each of these four 


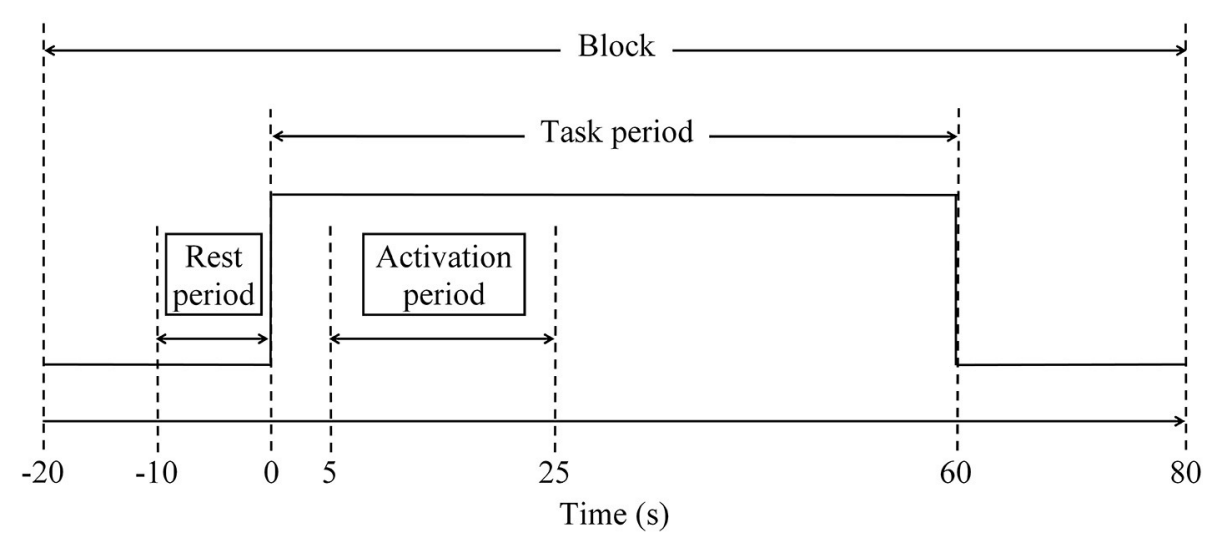

FIGURE 3 | Schematic diagram of a block to facilitate analysis. Each block consisted of 20-s pre-task rest period, 60-s task period and 20-s post-task period. Three categories of SVFT can be represented as three 100-s blocks.

fNIRS parameters, statistical significance was estimated using multiple comparisons between the three different groups at each category level, with two sets of independent two-sample $t$-tests-one without multiple testing correction and one with similar Bonferroni-Holm correction. Finally, to investigate the relationship between the MMSE score and various fNIRS parameters, correlation and simple linear regression analyses were performed using the MMSE score as a continuous independent variable.

\section{RESULTS}

\section{Sample Characteristics}

We excluded the data collected from one patient with moderate AD (CDR Score $=2$ ) as well as two left-handed participants (one each from $\mathrm{HC}$ and $\mathrm{MCI}$, as we were focusing only on righthanded participants). The final group of participants consisted of $31 \mathrm{HC}$ and 30 patients (MCI: 12; mild AD: 18) matched for age, sex, education, and handedness. Demographic information about age, gender and education level was collected (see Table 1).

\section{Behavioral Data}

The behavioral results (MMSE scores and the number of words generated in the three categories of SVFT) are summarized in Table 1 and illustrated in Figure 4. The number of comparisons for MMSE scores is three (for the three groups) and for SVFT is nine (3 groups $\times 3$ categories). There was a significant difference in MMSE score between the groups (see Table 1 and Figure 4A; $p$ : $\mathrm{HC}$ vs. $\mathrm{MCI}=0.0033, \mathrm{HC}$ vs. mild $\mathrm{AD}<0.0001, \mathrm{MCI}$ vs. mild $\mathrm{AD}=0.0012$ ). HC had the highest MMSE scores, followed by $\mathrm{MCI}$, then mild AD. Referring to Figure 4B, significant group differences in the number of words given in the "Fruits" category ( $p$ : HC vs. $\mathrm{MCI}=0.0081, \mathrm{HC}$ vs. mild $\mathrm{AD}<0.0001, \mathrm{MCI}$ vs. mild $\mathrm{AD}=0.0084)$ and the "Food" category ( $p: \mathrm{HC}$ vs. $\mathrm{MCI}=0.0094$, $\mathrm{HC}$ vs. mild $\mathrm{AD}<0.0001$, MCI vs. mild $\mathrm{AD}=0.0079$ ) were found. For the "Animals" category, the number of words given by HC was significantly higher than for mild AD $(p<0.0001)$. In comparison to MCI, HC produced more words, but it was not statistically significant ( $p=0.0217$ ). The number of words given by MCI was also higher than mild AD, but the difference was not statistically significant $(p=0.1669)$.

\section{fNIRS Data}

To characterize the fNIRS responses, we derived the following parameters, as shown in Figure 5:

(i) the number of activated measurement channels, $N_{\text {left } P F C / \text { right PFC }}$

(ii) the change in oxy-Hb concentration during the activation period, $\triangle o x y-H b_{\text {left } P F C / \text { right } P F C}$

(iii) the time taken to achieve the activation level, $t_{\text {left } P F C / \text { right } P F C}$

(iv) the slope in the first $5 \mathrm{~s}$ after task onset, $m_{\text {left } P F C / \text { right } P F C}$

As illustrated in Figure 5A, $N_{\text {left } P F C}$ was found to be higher (but not significantly so) than $N_{\text {right PFC }}$ in both HC and MCI. However, mild $\mathrm{AD}$ showed a higher $N_{\text {right } P F C}$, compared to the $N_{\text {left } P F C}$. The oxy-Hb signals measured in these activated measurement channels were then averaged across each group of participants to obtain an overall signal for the left and right PFC (see Figure 6).

Figure 5B shows the $\triangle o x y-H b_{\text {left } P F C / \text { right } P F C}$ for all groups. The highest $\triangle o x y-H b_{\text {left } P F C}$ was observed in MCI followed by $\mathrm{HC}$, while mild $\mathrm{AD}$ showed the lowest $\triangle o x y-H b_{\text {left PFC. A }}$ similar trend was also observed in the right PFC. However, in both the left and right PFC there were no significant differences between all three groups with respect to the level of activation. Nevertheless, all three groups demonstrated a similar trend, with higher $\triangle o x y-H b_{\text {right } P F C}$ compared to $\triangle o x y-H b_{\text {left } P F C}$, although differences were not statistically significant.

The $t_{\text {left } P F C / \text { right } P F C}$ was calculated and is illustrated in Figure 5C. When no multiple testing correction was used, the $t_{\text {left } P F C}$ by HC was shorter than MCI $(p=0.0047)$ and mild AD ( $p=0.0498)$. On the other hand, mild AD took a longer time than HC $(p=0.0469)$ and MCI $(p=0.0335)$ in the right PFC. The $m_{\text {left }}$ PFC/right PFC was also determined (see Figure 5D). Both $\mathrm{HC}$ and MCI showed steeper $m_{\text {right } P F C}$ when compared to mild 
TABLE 1 | Participants' demographic information and the pairwise Mann-Whitney U-test results.

\begin{tabular}{|c|c|c|c|c|c|c|}
\hline \multirow[b]{3}{*}{ Characteristic } & \multirow{3}{*}{$\begin{array}{l}\text { HC }(n=31) \\
\text { Mean (SD) }\end{array}$} & \multirow{3}{*}{$\begin{array}{l}\mathrm{MCI}(\mathrm{n}=12) \\
\text { Mean (SD) }\end{array}$} & \multirow{3}{*}{$\begin{array}{c}\text { mild AD }(n=18) \\
\text { Mean (SD) }\end{array}$} & \multicolumn{3}{|c|}{ Mann-Whitney U-test } \\
\hline & & & & \multicolumn{3}{|c|}{$p$-value $(r)$} \\
\hline & & & & HC vs. $\mathrm{MCl}$ & HC vs. mild AD & $\mathrm{MCl}$ vs. mild AD \\
\hline Age, years & $72.6(8.5)$ & $73.1(8.2)$ & 74.7 (10.0) & & & \\
\hline Gender, M/F & $19 / 12$ & $8 / 4$ & $12 / 6$ & & & \\
\hline Education level, P/S/T & $3 / 18 / 10$ & $1 / 7 / 4$ & $3 / 12 / 3$ & & & \\
\hline CDR rating & 0 & 0.5 & 1 & & & \\
\hline MMSE score & $28.7(1.5)$ & $26.0(3.1)$ & $21.2(3.6)$ & $0.0033^{\star \star}(0.4485)$ & $<0.0001^{\star \star}(0.7957)$ & $0.0012^{\star \star}(0.5896)$ \\
\hline \multicolumn{7}{|l|}{ SVFT, words } \\
\hline Fruits & $13.6(4.9)$ & $9.0(3.7)$ & $5.7(2.2)$ & $0.0081^{\star \star}(0.4036)$ & $<0.0001^{\star \star}(0.6934)$ & $0.0084^{* *}(0.4809)$ \\
\hline Food & $14.6(4.9)$ & $10.2(3.9)$ & $6.3(2.7)$ & $0.0094^{\star *}(0.3961)$ & $<0.0001^{\star \star}(0.7459)$ & $0.0079^{* *}(0.4851)$ \\
\hline Animals & $15.2(3.7)$ & $10.8(5.7)$ & $7.8(3.0)$ & $0.0217^{\star \star}(0.3500)$ & $<0.0001^{\star \star}(0.7304)$ & $0.1669(0.2524)$ \\
\hline
\end{tabular}

${ }^{\star *} p<0.05$ with Bonferroni-Holm correction.

HC, healthy control; MCl, mild cognitive impairment; mild AD, mild Alzheimer's disease; r, effect size; SD, standard deviation; M, male; F, female; P, primary; S, secondary; T, tertiary.

$\mathrm{AD}(p=0.0432$ and 0.0107 , respectively). In summary, HC used a shorter $t_{\text {left } P F C}$ than MCI and mild AD, and mild AD took longer $t_{\text {right } P F C}$ than $\mathrm{HC}$ and MCI, while $\mathrm{HC}$ and MCI demonstrated a steeper $m_{\text {right } P F C}$ than mild AD. However, the results described above were not significant when a Bonferroni-Holm correction was applied (number of comparisons is 3 groups $\times 4$ fNIRS parameters $=12$ ).

We also investigated, using simple linear regression, if there was any correlation between fNIRS parameters, i.e., $\quad N_{\text {left } P F C / \text { right PFC }}, \quad N_{\text {left } P F C / \text { right PFC, }}, t_{\text {left } P F C / \text { right } P F C}$ and

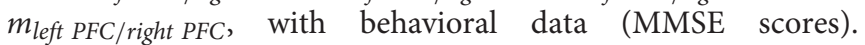
Interestingly, there was a moderate positive correlation $(R$ $\geq 0.5$ ) between one of the fNIRS parameters and the MMSE score in MCI, but not in the case of $\mathrm{HC}$ or mild AD. More specifically, in the left PFC, $\triangle o x y-H b_{\text {left } P F C}$ was moderately correlated to the MMSE score $(R=0.5886)$, as illustrated in Figure 7; Table 2 summarizes the results.

Finally, we noticed a large degree of inter-subject variation in fNIRS parameters, as shown in Figure 5. Such high variance not only makes the small group differences hard to distinguish statistically, but also results in a large overlap between the data from all three groups.

\section{DISCUSSION}

\section{Behavioral Data}

This study was designed to investigate the differences in prefrontal oxygenation between normal cognitive aging, MCI and mild $\mathrm{AD}$ using fNIRS. On a behavioral level, HC performed better than MCI, followed by mild AD in all three categories of SVFT, except for with the "Animals" category between HC and MCI; here, no significant difference was found between MCI and mild $\mathrm{AD}$. We also observed that participants, regardless of study group, were relatively faster in providing responses during the "Animals" category compared to the "Food" and "Fruits" categories. This finding might be due to all three groups being more familiar with the names of animals. This has been reported in previous studies, which have shown that naming is influenced by item frequency and familiarity (Patterson and Hodges, 1992; Lambon Ralph et al., 1998). It is possible that this difference between categories may be related to the varying degree of distinctive features among category members (Moss et al., 2002). This will not be elaborated upon further here, as it is not the main focus of the present study. Overall, these results were consistent with past studies that have utilized SVFT in various neuroimaging modalities (Fallgatter et al., 1997; Heinzel et al., 2013; Wagner et al., 2014; Yeung et al., 2016). This suggests that the SVFT is a reliable cortical activation task to be used in conjunction with fNIRS measurements. Here, it was observed that patients diagnosed with a greater degree of dementia, i.e., with higher CDR scores, gave repeated words more frequently. They had a tendency to forget which words they had already given, an attribute of a deteriorated right PFC where the working memory is for monitoring, or keeping immediate information on-line during tasks (Hayama and Rugg, 2009). The number of repetitions in SVFT and such attributes will not be discussed further here.

\section{fNIRS Data}

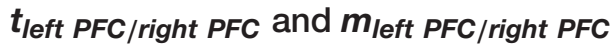

The results suggested that there were two fNIRS parameters that were significantly different between groups when the subjects were engaged in SVFT, only when no multiple testing correction was used. The first parameter is the $t_{\text {left } P F C / \text { right } P F C \text {. In the }}$ left PFC, HC took a shorter time in achieving the targeted activation level, compared to patients with MCI and mild AD. However, contrasting with the belief that MCI would have a faster activation than mild $\mathrm{AD}$, patients with mild $\mathrm{AD}$ actually took a shorter time. Conversely, with respect to the right PFC, MCI demonstrated the shortest time taken, followed by $\mathrm{HC}$ and mild AD. This is suggestive of the faster hemodynamic response in the right $\mathrm{PFC}$ of the MCI possibly being a compensatory response for the loss of the left PFC (Yeung et al., 2016). Taken separately, the poorer performance in SVFT and smaller 

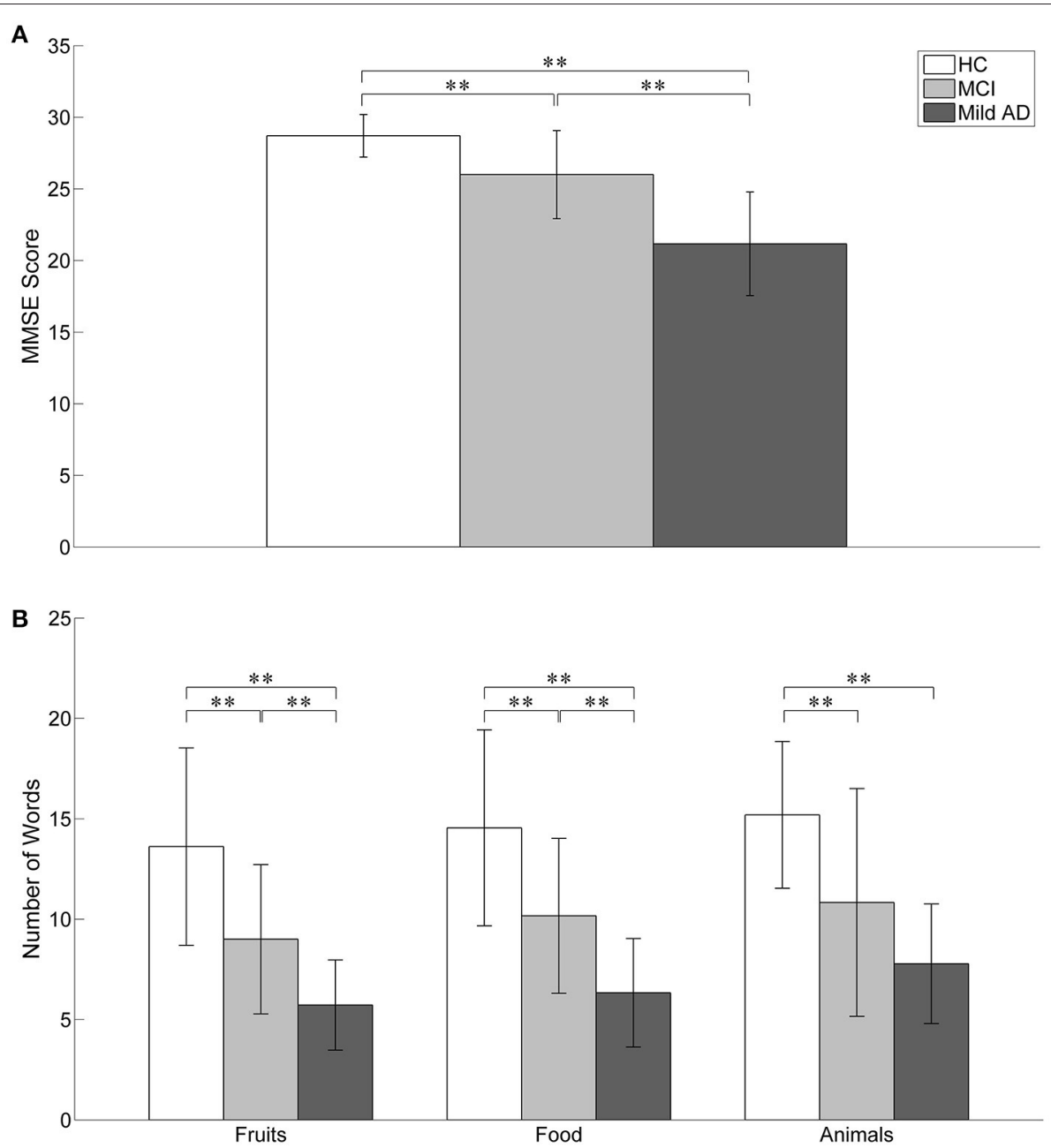

FIGURE 4 | The behavioral results. Statistical analysis was performed used two-sample $t$-test. ${ }^{* \star} p<0.05$ with Bonferroni-Holm correction. The error bars represent the standard deviations. (A) The MMSE score of $\mathrm{HC}$ was significantly higher than $\mathrm{MCl}$ and mild AD. MCl also achieved significant higher MMSE score as compared to mild AD. (B) In the SVFT, the performance was measured by the number of words given. Significant group differences between all three groups were found in the number of words given in "Fruits" and "Food" categories. For "Animals" category, the number of words given by HC was significantly higher than mild AD. In comparison to $\mathrm{MCl}, \mathrm{HC}$ produced more words but it was not statistically significant. The number of words given by $\mathrm{MCl}$ was also higher than mild AD but the difference was not significant.

activation in conjunction with the shorter time taken for left PFC activation in patients with mild AD might suggest that the compensatory mechanism is compromised. The second parameter examined was the $m_{\text {left } P F C / \text { right } P F C \text {. Experimental }}$ results showed that the $m_{\text {right } P F C}$ was significantly greater in MCI compared to mild $\mathrm{AD}$, further suggesting its importance to describing compensatory mechanisms. While the BonferroniHolm correction demonstrated that there were no significant differences, it is worthwhile explaining here the underlying mechanisms for these results.

Two possible explanations have been proposed for the underlying mechanism. The first explanation was consistent with that of the scaffolding theory of aging and cognition, in which additional circuitry is recruited to support declining brain function that has become inefficient (Park and Bischof, 2013).
This is commonly manifested in older adults that show increased contralateral right PFC recruitment for both working memory and episodic encoding (Reuter-Lorenz et al., 2000; Cabeza et al., 2002), which is consistent with our results. Such bilateral activation may be a form of interhemispheric interaction that has been claimed to be vital in neural compensatory mechanisms (Banich, 1998; Cabeza, 2002). Additionally, the results presented here imply that compensation and neuroplasticity might be present in the PFC of MCI, but not in mild AD. It has also been suggested that such compensatory ability might be reduced or lost in the progression of MCI toward $\mathrm{AD}$, as neurodegeneration suffered by $\mathrm{AD}$ patients is severe enough to halt natural compensation (Clement et al., 2013). This is supportive of the results presented here, where patients with mild $\mathrm{AD}$ tended to forget which words they had already given added to 


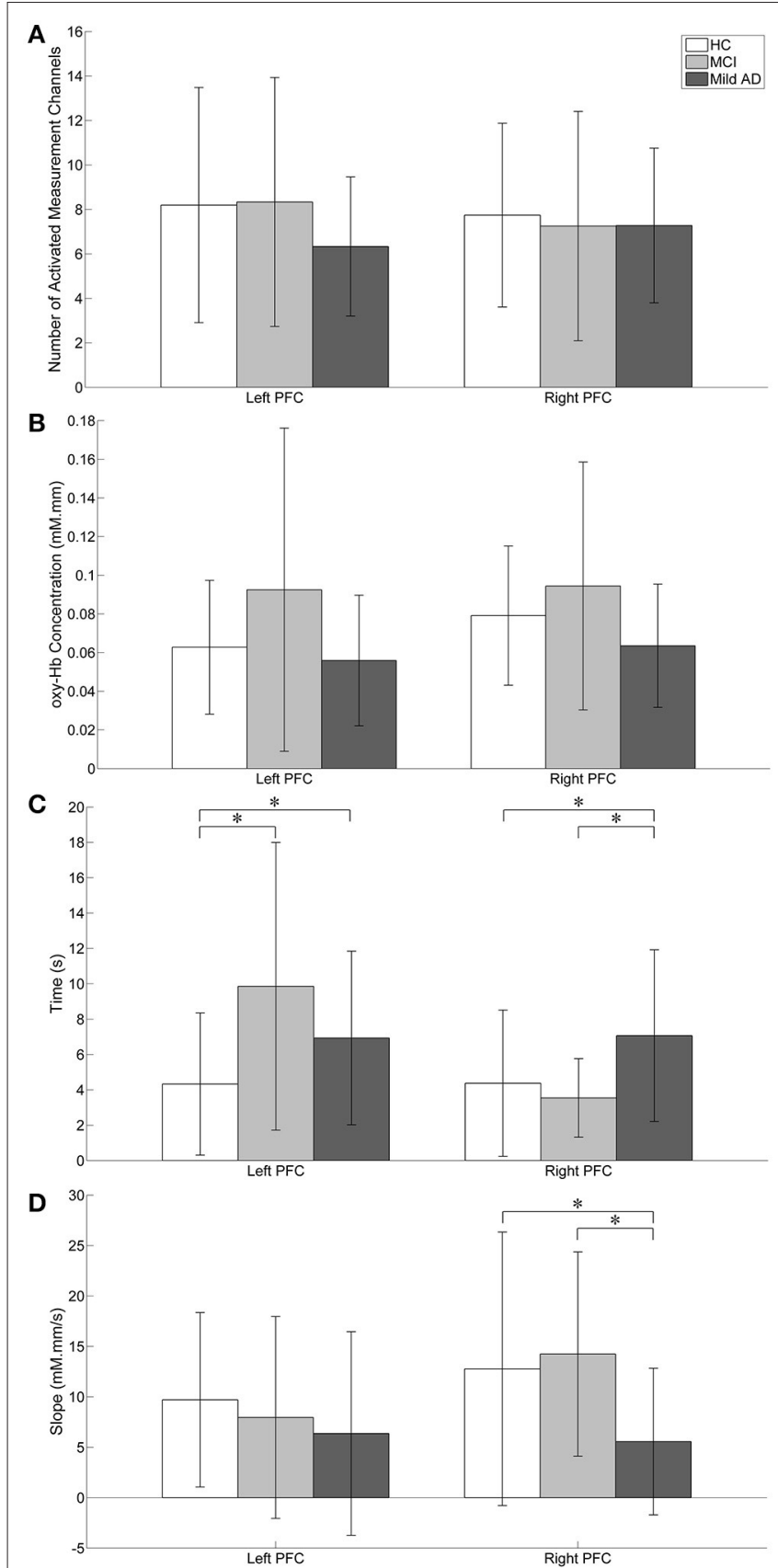

FIGURE 5 | Visual representation of fNIRS data. Statistical analysis was performed used two-sample $t$-test. ${ }^{*} p<0.05$ without multiple testing correction. The error bars represent the standard deviations. (A) The number of activated measurement channels: $N_{\text {left PFC/right PFC; there were no }}$ significant differences between groups in both the left and right PFC. (B) oxy- $\mathrm{Hb}$ concentration change during the activation period:

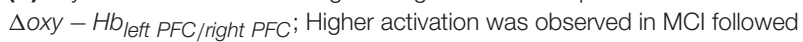
by $\mathrm{HC}$ while mild AD showed the least in both the left and right PFC. The right PFC was more activated than the left PFC in all groups. For both the left and right PFC, there were no significant group differences. (C) Time

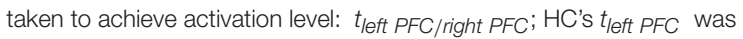
significantly shorter than $\mathrm{MCl}$. (D) Slope in the first $5 \mathrm{~s}$ after task onset:

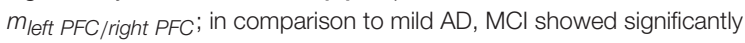
steeper $m_{\text {right } P F C \text {. }}$ the lack of activation of their right PFC, which is responsible for monitoring the immediate information during a task (Hayama and Rugg, 2009). This is further supported as the right PFC is specifically involved in semantic aspects of lexico-semantic processing (Joanette and Goulet, 1986).

We propose further, that the additional right $\mathrm{PFC}$ involvement and poorer memory performance can be explained using the inhibitory hypothesis. According to the inhibitory hypothesis, the non-dominant right PFC is normally suppressed by its dominant contra-lateral counterpart (Cox et al., 2015). Such transcallosal inhibition might be impaired with left PFC or anterior corpus callosum atrophy, which might result in extra recruitment of the right PFC. It has been found that additional non-dominant right PFC activity might reflect age-related changes in the brain and has been reported to be negatively correlated with memory performance (de Chastelaine et al., 2011). However, it has also been suggested that such disinhibition could reflect an attempted compensatory process, which is insufficient to fully compensate for age-related neurodegeneration. Our results point more toward the first explanation as we found that MCI, with right $\mathrm{PFC}$ activation relatively higher than both $\mathrm{HC}$ and mild $\mathrm{AD}$, actually performed better than the latter in the SVFT. Hence, we suggest that the identification of a compensatory role for the right PFC might offer a potential target area for neurorehabilitation (Cotelli et al., 2008). It is expected that in the future the residual plasticity in the right PFC of cognitively impaired patients, particularly those with MCI, might be effectively harnessed by neurorehabilitation and other interventional techniques. At this juncture, it is necessary to examine other parameters to identify differences in task-related activities across different populations.

\section{$N_{\text {left PFC/right PFC }}$}

Considering the number of activated fNIRS channels, there were no significant differences between both the right and left PFC for all three groups. The left PFC, which is responsible for semantic memory, was activated in the SVFT, as expected (Grossman et al., 2002). The activation of the right PFC, however, may be suggestive of participants being engaged in object imagery prior to recalling names during the task. In addition, it might also indicate ongoing monitoring of semantic information (Hayama and Rugg, 2009); the episodic memory located in the right PFC was engaged to ensure that participants did not repeat names during the task. As compared with $\mathrm{HC}$, a smaller region of right PFC activation was found in the other two groups (MCI and mild $\mathrm{AD}$ ). This could indicate the lack of a monitoring process, thus partially explaining poorer performance in SVFT in these groups compared to HC. However, this cannot explain the differences in SVFT performances between MCI and mild AD.

\section{$\Delta$ oxy $-H b_{\text {left PFC/right PFC }}$}

MCI demonstrated the greatest oxygenation levels of PFC activation, in both the left and right hemispheres, during SVFT, followed by HC, and mild AD. Despite not being statistically significant, this result might be of clinical significance. The result is in agreement with previous studies, utilizing various neuroimaging modalities, which have demonstrated similar 


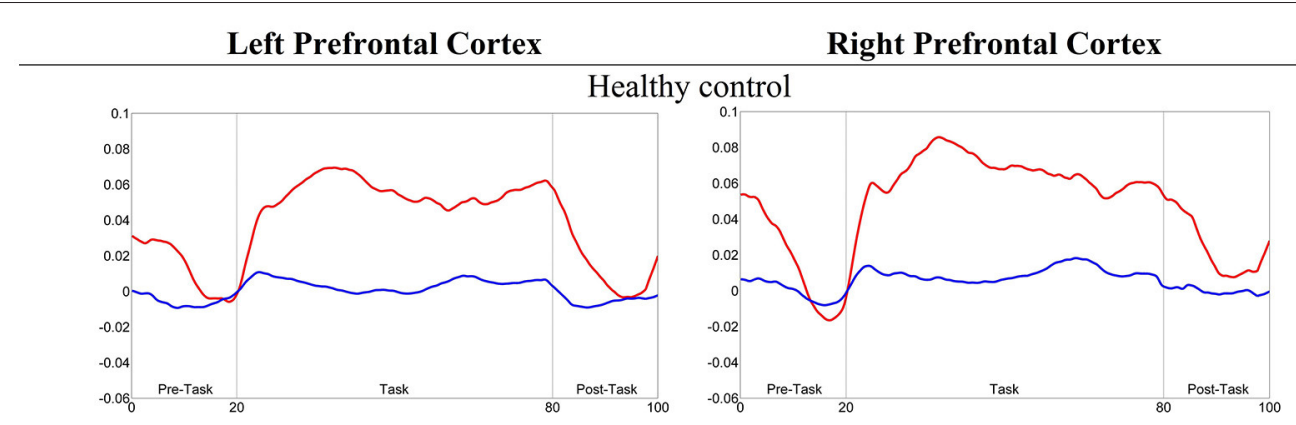

Mild cognitive impairment

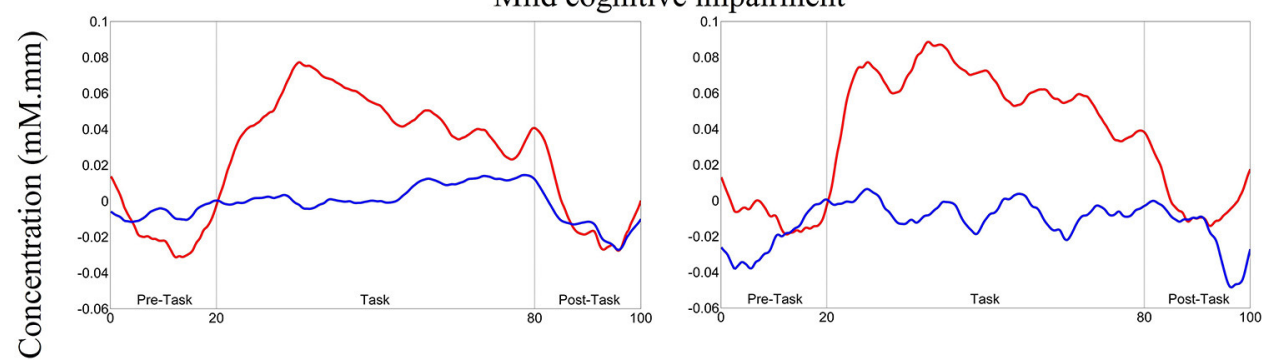

Mild Alzheimer's disease

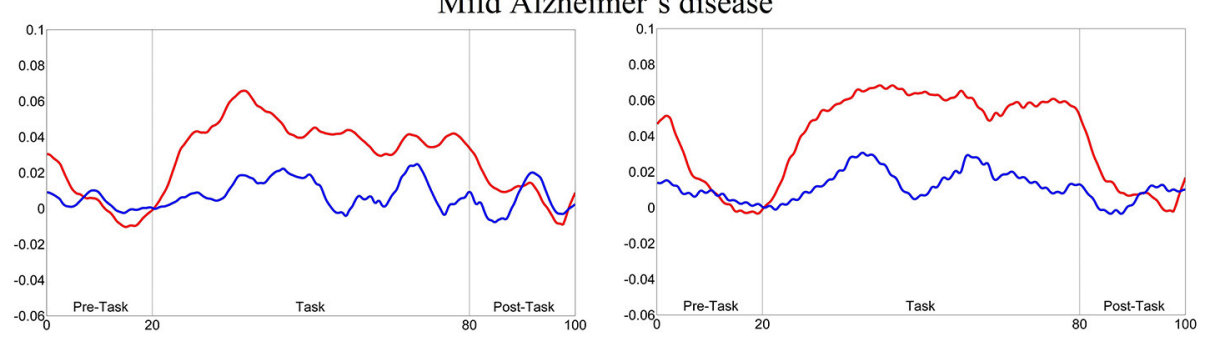

Time (s)

$-=$ oxy-Hb

$-=$ deoxy-Hb

FIGURE 6 | The overall oxy- and deoxy-Hb signals in the left and right PFC for all three groups.
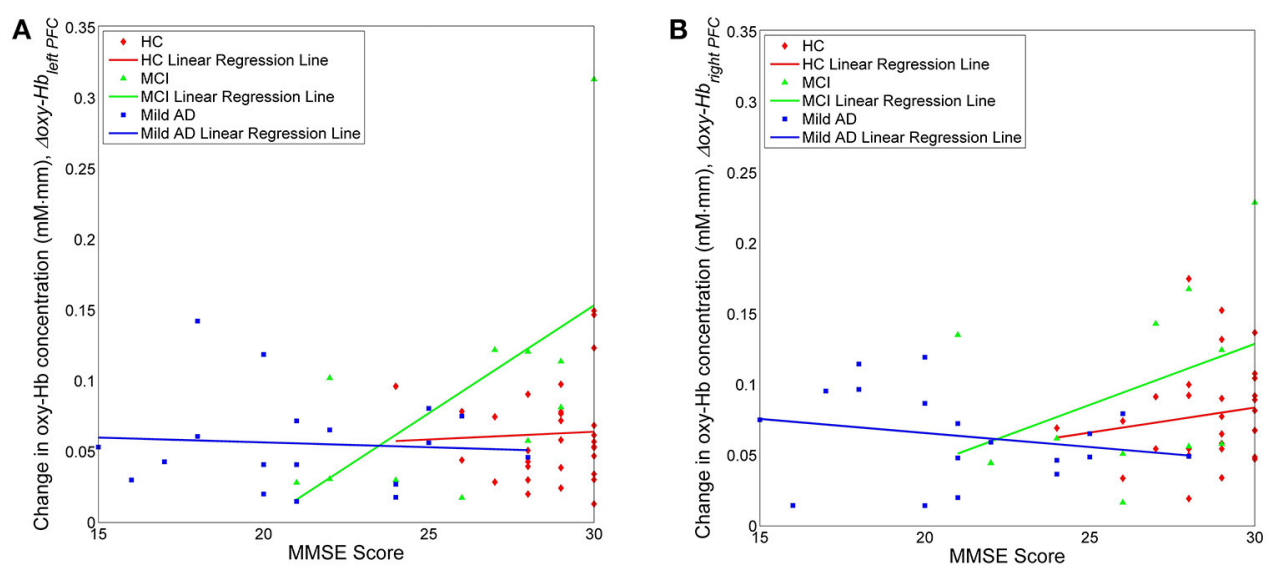

FIGURE 7 | Change in oxygenation level in the left (A) and right (B) PFC for all three groups against clinical measure, i.e., MMSE score. 
TABLE 2 | Pairwise correlation and simple linear regression results between fNIRS parameters, and MMSE score.

\begin{tabular}{|c|c|c|c|c|}
\hline & \multicolumn{2}{|c|}{ Left PFC } & \multicolumn{2}{|c|}{ Right PFC } \\
\hline & $m$ & $\mathbf{R}$ & $\boldsymbol{m}$ & $\mathbf{R}$ \\
\hline \multicolumn{5}{|c|}{ NUMBER OF ACTIVATED MEASUREMENT CHANNELS, } \\
\hline \multicolumn{5}{|c|}{$N_{\text {left PFC/right PFC }}$ vs. MMSE SCORE } \\
\hline $\mathrm{HC}$ & 0.1919 & 0.0540 & 0.3868 & 0.1393 \\
\hline $\mathrm{MCl}$ & -0.1154 & -0.0634 & 0.1731 & 0.1032 \\
\hline Mild AD & 0.2517 & 0.2914 & 0.0412 & 0.0429 \\
\hline \multicolumn{5}{|c|}{ oxy-Hb CONCENTRATION CHANGE DURING THE ACTIVATION PERIOD } \\
\hline \multicolumn{5}{|c|}{$\Delta o x y-H b_{\text {left PFC/right PFC }}$ vs. MMSE SCORE } \\
\hline $\mathrm{HC}$ & 0.0010 & 0.0478 & 0.0035 & 0.1467 \\
\hline $\mathrm{MCl}$ & 0.0152 & 0.5886 & 0.0086 & 0.4144 \\
\hline Mild AD & -0.0006 & -0.0732 & -0.0019 & -0.2258 \\
\hline \multicolumn{5}{|c|}{ TIME TAKEN TO ACHIEVE $25 \%$ OF PEAK ACTIVATION LEVEL, } \\
\hline \multicolumn{5}{|c|}{$t_{\text {left PFC/right PFC }}$ vs. MMSE Score } \\
\hline $\mathrm{HC}$ & -0.8478 & -0.3394 & -0.8567 & -0.3713 \\
\hline $\mathrm{MCl}$ & -0.4644 & -0.3340 & 0.4153 & 0.3143 \\
\hline Mild AD & 0.0961 & 0.0729 & -0.3606 & -0.2733 \\
\hline \multicolumn{5}{|c|}{ SLOPE IN THE FIRST 5 S AFTER TASK ONSET, } \\
\hline \multicolumn{5}{|c|}{$m_{\text {left PFC/right PFC }}$ vs. MMSE SCORE } \\
\hline $\mathrm{HC}$ & 0.0008 & 0.2040 & 0.0020 & 0.2926 \\
\hline $\mathrm{MCl}$ & 0.0007 & 0.2028 & -0.0001 & -0.0394 \\
\hline Mild AD & 0.0000 & -0.0026 & 0.0004 & 0.2663 \\
\hline
\end{tabular}

$m$, slope of the linear regression; $R$, correlation coefficient between the two parameters; $\mathrm{HC}$, healthy control; $\mathrm{MCl}$, mild cognitive impairment; mild AD, mild Alzheimer's disease.

findings (Johnson et al., 2000; Arai et al., 2006; Sperling, 2007; Driscoll et al., 2009; Woodard et al., 2009). As the dorsolateral PFC has been suggested to be associated with compensatory mechanisms (Erickson et al., 2007), the brain region-specific hyperactivation and hypoactivation observed in MCI and mild $\mathrm{AD}$, respectively, might indicate the presence of neural compensation in the former, and the inability to compensate in the latter (Prvulovic et al., 2005; Clement and Belleville, 2012). The differences in the level of activation across groups might also explain the differences in performance in SVFT, particularly in the left hemisphere (Fallgatter et al., 1997; Arai et al., 2006; Herrmann et al., 2008). However, contrary to current opinion, in the present study activation in the right PFC was greater compared to the left PFC, and was consistent between all the groups. This might be for the following reasons: (a) the activation of right PFC in monitoring cognitive processes during SVFT might implicate a greater role compared to the left PFC; (b) the difference might be due to methodological differences, including the differences in recruitment strategies, brain region investigated and variations in research design and measurements used to describe the outcome. More specifically, the variations in research design refer to different assessment tools, inclusion and exclusion criteria (Fallgatter et al., 1997; Yeung et al., 2016), the numbers of groups (e.g., between normal aging and AD; Herrmann et al., 2008) or between normal aging and MCI (Yeung et al., 2016), and the focus on a brain region (e.g., frontal and parietal lobe). Similarly, differences in the outcomes measured might exert a significant impact on the results, such as separate analyses of oxy-Hb and deoxy-Hb (Herrmann et al., 2008) and different baseline-corrected values (Fallgatter et al., 1997). The high variance observed here might be due to placement of the probes i.e., the position of the optodes relative to the skin (Strangman et al., 2003). Inter-subject anatomical variability, such as the thicknesses of the skull and the cerebrospinal fluid layers could have also caused the large variation in the fNIRS measurements (Okada and Delpy, 2003).

\section{Moderate Positive Correlation between $\Delta$ oxy $-\boldsymbol{H} b_{\text {left PFC }}$ and MMSE Score in $\mathrm{MCl}$}

Finally, we propose an explanation for the moderately positive ( $R=0.5886)$ linear relationship between the oxygenation level in the left PFC and the MMSE score, which was found only in MCI subjects, but not in $\mathrm{HC}$ and mild $\mathrm{AD}$. This result reflects the fact that increasing activation actually contributed to the cognitive status in MCI, while both $\mathrm{HC}$ and mild $\mathrm{AD}$ did not show a similar trend. We suggest that in $\mathrm{HC}$, the behavioral ceiling effect might have been achieved, as represented by the maximum score in MMSE (30) and this does not necessarily imply the presence of a parallel ceiling in brain activation (continuous increases in left PFC activation) (Hagenbeek et al., 2007). This might also indicate that the neural network is intact in HC. In addition, MMSE might be a relatively easy task for $\mathrm{HC}$ and the maximum score of 30 points may, therefore, not be a sensitive measure of cognitive status. MMSE is not designed to measure the cognitive ability of a healthy person. However, cognitive status in MCI is well below the ceiling. The pathogenesis of $\mathrm{AD}$ is characterized pathologically by brain accumulation of amyloid $\beta$-protein $(A \beta)$ in the early stages (Jack et al., 2013) and $A \beta$ is thought to be the cause of neuronal dysfunction in AD (Palop and Mucke, 2010), which necessitates neural activity. Previous studies have reported that, relative to younger people or older people without $\mathrm{A} \beta$, both cognitively normal older people with $A \beta$ deposition (Mormino et al., 2012) and MCI patients (Dickerson et al., 2004) exhibit higher neuronal activity during cognitive task performance. This phenomenon might be evidence of functional compensation keeping older people with $\mathrm{A} \beta$ and MCI patients cognitively stable. In agreement with these results, the increase in oxygenation levels might represent an attempted compensatory response, and hence it is proportionate to the improvement in cognitive status in MCI. However, it is not possible to draw any conclusions with respect to the interpretation due to the small MCI sample size here. Meanwhile, for mild $\mathrm{AD}$, no linear relationship was found between the oxygenation levels in the left PFC and the MMSE score. The degree of $A \beta$ deposition in the brain might reduce neural efficiency, which eventually causes progression to a more severe stage of AD (Landau et al., 2012). In such a situation, it is possible that patients eventually decline cognitively as any compensatory ability has been compromised (O’Brien et al., 2010). So, when patients progress into mild $\mathrm{AD}$, their neural compensation ability might have been weakened or the neural networks might have been compromised to the point where higher oxygenation levels coupled with compensatory mechanisms are no longer enough to maintain cognitive status, unlike with MCI. Although 
the explanations above might fit with the observations, it is important to note that various non-neural factors might confound such interpretations; disrupted neurovascular coupling that is associated with pathological conditions e.g., aging and disease (Buckner et al., 2000; D'Esposito et al., 2003) is one of the many factors. Other factors include alterations in perfusion and metabolism (El Fakhri et al., 2003), and vascular physiology (Mueggler et al., 2002). It has also been reported that employing different verbal memory strategies led to different patterns of cortical activation (Logan et al., 2002). A final factor that might have influenced PFC activation is the administration of medication e.g., cholinergic stimulation (Rombouts et al., 2002) and donepezil (McGeown et al., 2010).

\section{Limitation}

Subtypes of MCI need to be considered: a clinical presentation with memory impairment is characterized as amnestic MCI (aMCI), whereas the absence of memory impairment with the presence of impairment in one or more non-memory cognitive domains is characterized as non-amnestic MCI (naMCI). Furthermore, these subtypes can be further narrowed down into single and multi-domain impairments. It has been suggested that aMCI has a higher likelihood of progressing into $\mathrm{AD}$, while naMCI is prone to developing into non-AD dementia (Petersen et al., 2009). Since the present study accessed a relatively small number of MCI patients, no attempt was made to exclude patients on the basis of other comorbidities. To substantiate the findings, research with a larger sample size might help ensure that participants with secondary comorbidities can be excluded. In addition, such a study could ensure that participants with different subtypes of MCI are assessed separately.

\section{CONCLUSION}

It was found that $\mathrm{HC}$ took a shorter time to achieve the targeted activation level in the left PFC compared to MCI and mild $\mathrm{AD}$, while mild $\mathrm{AD}$ took a longer time than $\mathrm{HC}$ and $\mathrm{MCI}$ in the

\section{REFERENCES}

Alzheimer's Association (2016). 2016 Alzheimer's disease facts and figures. Alzheimer's Dement. 12, 459-509. doi: 10.1016/j.jalz.2016.03.001

Arai, H., Takano, M., Miyakawa, K., Ota, T., Takahashi, T., Asaka, H., et al. (2006). A quantitative near-infrared spectroscopy study: a decrease in cerebral hemoglobin oxygenation in Alzheimer's disease and mild cognitive impairment. Brain Cogn. 61, 189-194. doi: 10.1016/j.bandc.2005.12.012

Baazaoui, N., Flory, M., and Iqbal, K. (2017). Synaptic compensation as a probable cause of prolonged mild cognitive impairment in Alzheimer's disease: implications from a transgenic mouse model of the disease. J. Alzheimers Dis. 56, 1385-1401. doi: 10.3233/JAD-160845

Banich, M. T. (1998). The missing link: the role of interhemispheric interaction in attentional processing. Brain Cogn. 36, 128-157. doi: 10.1006/brcg.1997.0950

Barnes, J., Godbolt, A. K., Frost, C., Boyes, R. G., Jones, B. F., Scahill, R. I., et al. (2007). Atrophy rates of the cingulate gyrus and hippocampus in $\mathrm{AD}$ and FTLD. Neurobiol. Aging 28, 20-28. doi: 10.1016/j.neurobiolaging.2005.11.012

Buckner, R. L., Snyder, A. Z., Sanders, A. L., Raichle, M. E., and Morris, J. C. (2000). Functional brain imaging of young, nondemented, and demented older adults. J. Cogn. Neurosci. 12(Suppl. 2), 24-34. doi: 10.1162/089892900564046 right PFC. In addition, a steeper slope of activation was found in the right PFC of patients with MCI compared to HC and mild $\mathrm{AD}$. The right $\mathrm{PFC}$ was particularly recruited in compensatory activity, which could be explained by the scaffolding theory of aging and cognition, and the inhibitory theory. Our results demonstrated, by using fNIRS, that compensation and neuroplasticity in the form of hyperactivation might be present in the PFC of MCI, but not in mild AD. Compensatory mechanisms might, therefore, have been compromised in mild AD. Time taken and the slope of activation were identified as key parameters of neuronal compensatory mechanisms, although the results presented here were not statistically significant after Bonferroni-Holm corrections. Future studies should look at these parameters individually. A moderately positive correlation between the oxygenation level in the left PFC and MMSE score was also found uniquely in MCI subjects. Longitudinal studies would be helpful in confirming whether task-elicited hyperactivation in MCI and hypoactivation in mild AD do indeed reflect the presence of compensatory mechanisms and the inability to compensate, respectively. If they do, future studies with a larger sample size could be directed toward investigating these fNIRS parameters as potential prognostic biomarkers of MCI and mild AD progression.

\section{AUTHOR CONTRIBUTIONS}

TT, EE, and SS designed the study. KY, WU, NN, PC, SC, and HY acquired the data. KY, WU, MK, and TT analyzed the data. KY, WU, and TT wrote the article, which all authors reviewed and approved for publication.

\section{FUNDING}

This work was partially supported by MARA [Grant no: MARA/UNI.1/33/06/02/15(4)] and the Ministry of Higher Education, Malaysia under Higher Institution Centre of Excellence (HiCOE) scheme to CISIR, UTP.

Cabeza, R. (2002). Hemispheric asymmetry reduction in older adults: the HAROLD model. Psychol. Aging 17, 85-100. doi: 10.1037/0882-7974.17.1.85

Cabeza, R., Anderson, N. D., Locantore, J. K., and McIntosh, A. R. (2002). Aging gracefully: compensatory brain activity in high-performing older adults. Neuroimage 17, 1394-1402. doi: 10.1006/nimg.2002.1280

Clement, F., and Belleville, S. (2012). Effect of disease severity on neural compensation of item and associative recognition in mild cognitive impairment. J. Alzheimers Dis. 29, 109-123. doi: 10.3233/JAD-2012-110426

Clement, F., Gauthier, S., and Belleville, S. (2013). Executive functions in mild cognitive impairment: emergence and breakdown of neural plasticity. Cortex 49, 1268-1279. doi: 10.1016/j.cortex.2012.06.004

Cotelli, M., Manenti, R., Cappa, S. F., Zanetti, O., and Miniussi, C. (2008). Transcranial magnetic stimulation improves naming in Alzheimer disease patients at different stages of cognitive decline. Eur. J. Neurol. 15, 1286-1292. doi: 10.1111/j.1468-1331.2008.02202.x

Cox, S. R., Bastin, M. E., Ferguson, K. J., Allerhand, M., Royle, N. A., Maniega, S. M., et al. (2015). Compensation or inhibitory failure? Testing hypotheses of age-related right frontal lobe involvement in verbal memory ability using structural and diffusion MRI. Cortex 63, 4-15. doi: 10.1016/j.cortex.2014.0 8.001 
Cui, X. (2011). Realtime Feedback from NIRS Recording. Available online at: http://www.alivelearn.net/?p=1263

Cui, X., Bray, S., Bryant, D. M., Glover, G. H., and Reiss, A. L. (2011). A quantitative comparison of NIRS and fMRI across multiple cognitive tasks. Neuroimage 54, 2808-2821. doi: 10.1016/j.neuroimage.2010.10.069

Dannhauser, T. M., Shergill, S. S., Stevens, T., Lee, L., Seal, M., Walker, R. W., et al. (2008). An fMRI study of verbal episodic memory encoding in amnestic mild cognitive impairment. Cortex 44, 869-880. doi: 10.1016/j.cortex.2007.04.005

de Chastelaine, M., Wang, T. H., Minton, B., Muftuler, L. T., and Rugg, M. D. (2011). The effects of age, memory performance, and callosal integrity on the neural correlates of successful associative encoding. Cereb. Cortex 21, 2166-2176. doi: 10.1093/cercor/bhq294

D’Esposito, M., Deouell, L. Y., and Gazzaley, A. (2003). Alterations in the BOLD fMRI signal with ageing and disease: a challenge for neuroimaging. Nat. Rev. Neurosci. 4, 863-872. doi: 10.1038/nrn1246

Dickerson, B. C., Salat, D. H., Bates, J. F., Atiya, M., Killiany, R. J., Greve, D. N., et al. (2004). Medial temporal lobe function and structure in mild cognitive impairment. Ann. Neurol. 56, 27-35. doi: 10.1002/ana.20163

Driscoll, T., Kim, H.-T., Chae, B.-G., Kim, B.-J., Lee, Y.-W., Jokerst, N. M., et al. (2009). Memory metamaterials. Science 325, 1518-1521. doi: $10.1126 /$ science. 1176580

Ehlis, A.-C., Schneider, S., Dresler, T., and Fallgatter, A. J. (2014). Application of functional near-infrared spectroscopy in psychiatry. Neuroimage 85, 478-488. doi: 10.1016/j.neuroimage.2013.03.067

El Fakhri, G., Kijewski, M. F., Johnson, K. A., Syrkin, G., Killiany, R. J., Becker, J. A., et al. (2003). MRI-guided SPECT perfusion measures and volumetric MRI in prodromal Alzheimer disease. Arch. Neurol. 60, 1066-1072. doi: 10.1001/archneur.60.8.1066

Erickson, K. I., Colcombe, S. J., Wadhwa, R., Bherer, L., Peterson, M. S., Scalf, P. E., et al. (2007). Training-induced functional activation changes in dual-task processing: an FMRI study. Cereb. Cortex 17, 192-204. doi: 10.1093/cercor/bhj137

Fallgatter, A., Roesler, M., Sitzmann, A., Heidrich, A., Mueller, T., and Strik, W. (1997). Loss of functional hemispheric asymmetry in Alzheimer's dementia assessed with near-infrared spectroscopy. Cogn. Brain Res. 6, 67-72. doi: 10.1016/S0926-6410(97)00016-5

Ferrari, M., and Quaresima, V. (2012). A brief review on the history of human functional near-infrared spectroscopy (fNIRS) development and fields of application. Neuroimage 63, 921-935. doi: 10.1016/j.neuroimage.2012.03.049

Folstein, M. F., Folstein, S. E., and McHugh, P. R. (1975). "Mini-mental state": a practical method for grading the cognitive state of patients for the clinician. J. Psychiatr. Res. 12, 189-198. doi: 10.1016/0022-3956(75)90026-6

Gauthier, S., Reisberg, B., Zaudig, M., Petersen, R. C., Ritchie, K., Broich, K., et al. (2006). Mild cognitive impairment. Lancet 367, 1262-1270. doi: $10.1016 / \mathrm{S} 0140-6736(06) 68542-5$

Grossman, M., Smith, E. E., Koenig, P., Glosser, G., DeVita, C., Moore, P., et al. (2002). The neural basis for categorization in semantic memory. Neuroimage 17, 1549-1561. doi: 10.1006/nimg.2002.1273

Gutierrez-Sigut, E., Payne, H., and MacSweeney, M. (2015). Investigating language lateralization during phonological and semantic fluency tasks using functional transcranial Doppler sonography. Laterality 20, 49-68. doi: 10.1080/1357650X.2014.914950

Hagenbeek, R. E., Rombouts, S. A. R. B., Veltman, D. J., Van Strien, J. W., Witter, M. P., Scheltens, P., et al. (2007). Nonlinear changes in brain activity during continuous word repetition: an event-related multiparametric functional $\mathrm{mr}$ imaging study. Am. J. Neuroradiol. 28, 1715-1721. doi: 10.3174/ajnr.A0632

Hayama, H. R., and Rugg, M. D. (2009). Right dorsolateral prefrontal cortex is engaged during post-retrieval processing of both episodic and semantic information. Neuropsychologia 47, 2409-2416. doi: 10.1016/j.neuropsychologia.2009.04.010

Heinzel, S., Haeussinger, F. B., Hahn, T., Ehlis, A.-C., Plichta, M. M., and Fallgatter, A. J. (2013). Variability of (functional) hemodynamics as measured with simultaneous fNIRS and fMRI during intertemporal choice. Neuroimage 71, 125-134. doi: 10.1016/j.neuroimage.2012.12.074

Herrmann, M. J., Langer, J. B., Jacob, C., Ehlis, A.-C., and Fallgatter, A. J. (2008). Reduced prefrontal oxygenation in Alzheimer disease during verbal fluency tasks. Am. J. Geriatr. Psychiatry 16, 125-135. doi: 10.1097/JGP.0b013e3180cc1fbc
Ishii-Takahashi, A., Takizawa, R., Nishimura, Y., Kawakubo, Y., Kuwabara, H., Matsubayashi, J., et al. (2014). Prefrontal activation during inhibitory control measured by near-infrared spectroscopy for differentiating between autism spectrum disorders and attention deficit hyperactivity disorder in adults. Neuroimage Clin. 4, 53-63. doi: 10.1016/j.nicl.2013.10.002

Jack, C. R. Jr., Knopman, D. S., Jagust, W. J., Petersen, R. C., Weiner, M. W., Aisen, P. S., et al. (2013). Tracking pathophysiological processes in Alzheimer's disease: an updated hypothetical model of dynamic biomarkers. Lancet Neurol. 12, 207-216. doi: 10.1016/S1474-4422(12)70291-0

Joanette, Y., and Goulet, P. (1986). Criterion-specific reduction of verbal fluency in right brain-damaged right-handers. Neuropsychologia 24, 875-879. doi: 10.1016/0028-3932(86)90087-4

Johnson, S. C., Saykin, A. J., Baxter, L. C., Flashman, L. A., Santulli, R. B., McAllister, T. W., et al. (2000). The relationship between fMRI activation and cerebral atrophy: comparison of normal aging and Alzheimer disease. Neuroimage 11, 179-187. doi: 10.1006/nimg.1999.0530

Johnson, S. C., Schmitz, T. W., Moritz, C. H., Meyerand, M. E., Rowley, H. A., Alexander, A. L., et al. (2006). Activation of brain regions vulnerable to Alzheimer's disease: the effect of mild cognitive impairment. Neurobiol. Aging 27, 1604-1612. doi: 10.1016/j.neurobiolaging.2005.09.017

Karantzoulis, S., and Galvin, J. E. (2014). Distinguishing Alzheimer's disease from other major forms of dementia. Expert Rev. Neurother. 11, 1579-1591. doi: 10.1586/ern.11.155

Klem, G. H., Luders, H. O., Jasper, H. H., and Elger, C. (1999). The ten-twenty electrode system of the International Federation. The international federation of clinical neurophysiology. Electroencephalogr. Clin. Neurophysiol. Suppl. 52, $3-6$.

Lambon Ralph, M. A., Graham, K. S., Ellis, A. W., and Hodges, J. R. (1998). Naming in semantic dementia-what matters? Neuropsychologia 36, 775-784. doi: 10.1016/S0028-3932(97)00169-3

Landau, S. M., Mintun, M. A., Joshi, A. D., Koeppe, R. A., Petersen, R. C., Aisen, P. S., et al. (2012). Amyloid deposition, hypometabolism, and longitudinal cognitive decline. Ann. Neurol. 72, 578-586. doi: 10.1002/ana.23650

Li, T., Gong, H., and Luo, Q. (2011). Visualization of light propagation in visible Chinese human head for functional near-infrared spectroscopy. J. Biomed. Opt. 16, 045001. doi: 10.1117/1.3567085

Logan, J. M., Sanders, A. L., Snyder, A. Z., Morris, J. C., and Buckner, R. L. (2002). Under-recruitment and nonselective recruitment: dissociable neural mechanisms associated with aging. Neuron 33, 827-840. doi: 10.1016/S0896-6273(02)00612-8

Luu, S., and Chau, T. (2009). Decoding subjective preference from singletrial near-infrared spectroscopy signals. J. Neural Eng. 6:016003. doi: $10.1088 / 1741-2560 / 6 / 1 / 016003$

Magni, E., Binetti, G., Bianchetti, A., Rozzini, R., and Trabucchi, M. (1996). MiniMental State Examination: a normative study in Italian elderly population. Eur. J. Neurol. 3, 198-202. doi: 10.1111/j.1468-1331.1996.tb00423.x

Maki, A., Yamashita, Y., Ito, Y., Watanabe, E., Mayanagi, Y., and Koizumi, H. (1995). Spatial and temporal analysis of human motor activity using noninvasive NIR topography. Med. Phys. 22, 1997-2005. doi: 10.1118/1.597496

Mandzia, J. L., McAndrews, M. P., Grady, C. L., Graham, S. J., and Black, S. E. (2009). Neural correlates of incidental memory in mild cognitive impairment: an fMRI study. Neurobiol. Aging 30, 717-730. doi: 10.1016/j.neurobiolaging.2007.08.024

Marner, L., Nyengaard, J. R., Tang, Y., and Pakkenberg, B. (2003). Marked loss of myelinated nerve fibers in the human brain with age. J. Comp. Neurol. 462, 144-152. doi: 10.1002/cne.10714

McGeown, W. J., Shanks, M. F., Forbes-McKay, K. E., Waiter, G. D., Elrick, I., Venneri, M. G., et al. (2010). Established donepezil treatment modulates task relevant regional brain activation in early Alzheimer's disease. Curr. Alzheimer Res. 7, 415-427. doi: 10.2174/156720510791383877

McNab, F., and Klingberg, T. (2008). Prefrontal cortex and basal ganglia control access to working memory. Nat. Neurosci. 11, 103-107. doi: 10.1038/nn2024

Mormino, E. C., Brandel, M. G., Madison, C. M., Marks, S., Baker, S. L., and Jagust, W. J. (2012). A deposition in aging is associated with increases in brain activation during successful memory encoding. Cereb. Cortex 22, 1813-1823. doi: $10.1093 /$ cercor/bhr255

Morris, J. C. (1993). The Clinical Dementia Rating (CDR): current version and scoring rules. Neurology 43, 2412-2414. doi: 10.1212/WNL.43.11.2412-a 
Moss, H. E., Tyler, L. K., Devlin, J. T., Forde, E., and Humphreys, G. (2002). "The emergence of category-specific deficits in a distributed semantic system," Category-Specificity in Brain and Mind, eds E. Forde and G. Humphreys (Hove: Psychology Press), 115-148.

Mueggler, T., Sturchler-Pierrat, C., Baumann, D., Rausch, M., Staufenbiel, M., and Rudin, M. (2002). Compromised hemodynamic response in amyloid precursor protein transgenic mice. J. Neurosci. 22, 7218-7224.

Nho, K., Risacher, S. L., Crane, P. K., DeCarli, C., Glymour, M. M., Habeck, C., et al. (2012). Voxel and surface-based topography of memory and executive deficits in mild cognitive impairment and Alzheimer's disease. Brain Imaging Behav. 6, 551-567. doi: 10.1007/s11682-012-9203-2

O’Brien, J. L., O'Keefe, K. M., LaViolette, P. S., DeLuca, A. N., Blacker, D., Dickerson, B. C., et al. (2010). Longitudinal fMRI in elderly reveals loss of hippocampal activation with clinical decline. Neurology 74, 1969-1976. doi: 10.1212/WNL.0b013e3181e3966e

Okada, E., and Delpy, D. T. (2003). Near-infrared light propagation in an adult head model. II. Effect of superficial tissue thickness on the sensitivity of the near-infrared spectroscopy signal. Appl. Opt. 42, 2915-2922. doi: 10.1364/AO.42.002915

Palop, J. J., and Mucke, L. (2010). Amyloid- $\beta$ induced neuronal dysfunction in Alzheimer's disease: from synapses toward neural networks. Nat. Neurosci. 13, 812-818. doi: $10.1038 / \mathrm{nn} .2583$

Park, D. C., and Bischof, G. N. (2013). The aging mind: neuroplasticity in response to cognitive training. Dialogues Clin. Neurosci. 15, 109-119.

Patterson, K., and Hodges, J. R. (1992). Deterioration of word meaning: implications for reading. Neuropsychologia 30, 1025-1040. doi: 10.1016/0028-3932(92)90096-5

Perry, R. J., Watson, P., and Hodges, J. R. (2000). The nature and staging of attention dysfunction in early (minimal and mild) Alzheimer's disease: relationship to episodic and semantic memory impairment. Neuropsychologia 38, 252-271. doi: 10.1016/S0028-3932(99)00079-2

Petersen, R. C. (2009). Early diagnosis of Alzheimer's disease: is MCI too late? Curr. Alzheimer Res. 6, 324-330. doi: 10.2174/156720509788929237

Petersen, R. C., Roberts, R. O., Knopman, D. S., Boeve, B. F., Geda, Y. E., Ivnik, R. J., et al. (2009). Mild cognitive impairment: ten years later. Arch. Neurol. 66, 1447-1455. doi: 10.1001/archneurol.2009.266

Price, C. J., and Friston, K. J. (2002). Degeneracy and cognitive anatomy. Trends Cogn. Sci. 6, 416-421. doi: 10.1016/S1364-6613(02)01976-9

Prvulovic, D., Van de Ven, V., Sack, A. T., Maurer, K., and Linden, D. E. (2005). Functional activation imaging in aging and dementia. Psychiatry Res. 140, 97-113. doi: 10.1016/j.pscychresns.2005.06.006

Ranger, M., Johnston, C. C., Limperopoulos, C., Rennick, J. E., and du Plessis, A. J. (2011). Cerebral near-infrared spectroscopy as a measure of nociceptive evoked activity in critically ill infants. Pain Res. Manag. 16, 331-336. doi: 10.1155/2011/891548

Reuter-Lorenz, P. A., Jonides, J., Smith, E. E., Hartley, A., Miller, A., Marshuetz, C., et al. (2000). Age differences in the frontal lateralization of verbal and spatial working memory revealed by PET. J. Cogn. Neurosci. 12, 174-187. doi: 10.1162/089892900561814

Rockwood, K., Strang, D., MacKnight, C., Downer, R., and Morris, J. C. (2000). Interrater reliability of the Clinical Dementia Rating in a multicenter trial. J. Am. Geriatr. Soc. 48, 558-559. doi: 10.1111/j.1532-5415.2000.tb05004.x

Rombouts, S., Barkhof, F., van Meel, C. S., and Scheltens, P. (2002). Alterations in brain activation during cholinergic enhancement with rivastigmine in Alzheimer's disease. J. Neurol. Neurosurg. Psychiatry 73, 665-671. doi: 10.1136/jnnp.73.6.665

Salat, D. H., Kaye, J. A., and Janowsky, J. S. (2001). Selective preservation and degeneration within the prefrontal cortex in aging and Alzheimer disease. Arch. Neurol. 58, 1403-1408. doi: 10.1001/archneur.58.9.1403
Sato, H., Kiguchi, M., Maki, A., Fuchino, Y., Obata, A., Yoro, T., et al. (2006). Within-subject reproducibility of near-infrared spectroscopy signals in sensorimotor activation after 6 months. J. Biomed. Opt. 11, 014021. doi: $10.1117 / 1.2166632$

Scarmeas, N., Zarahn, E., Anderson, K. E., Hilton, J., Flynn, J., Van Heertum, R. L., et al. (2003). Cognitive reserve modulates functional brain responses during memory tasks: a PET study in healthy young and elderly subjects. Neuroimage 19, 1215-1227. doi: 10.1016/S1053-8119(03)00074-0

Shi, F., Liu, B., Zhou, Y., Yu, C., and Jiang, T. (2009). Hippocampal volume and asymmetry in mild cognitive impairment and Alzheimer's disease: metaanalyses of MRI studies. Hippocampus 19, 1055-1064. doi: 10.1002/hipo.20573

Sperling, R. (2007). Functional MRI studies of associative encoding in normal aging, mild cognitive impairment, and Alzheimer's disease. Ann. N. Y. Acad. Sci. 1097, 146-155. doi: 10.1196/annals.1379.009

Strangman, G. E., Li, Z., and Zhang, Q. (2013). Depth sensitivity and sourcedetector separations for near infrared spectroscopy based on the Colin27 brain template. PLoS ONE 8:e66319. doi: 10.1371/journal.pone.0066319

Strangman, G., Franceschini, M. A., and Boas, D. A. (2003). Factors affecting the accuracy of near-infrared spectroscopy concentration calculations for focal changes in oxygenation parameters. Neuroimage 18, 865-879. doi: 10.1016/S1053-8119(03)00021-1

Sutoko, S., Sato, H., Maki, A., Kiguchi, M., Hirabayashi, Y., Atsumori, H., et al. (2016). Tutorial on platform for optical topography analysis tools. Neurophotonics 3:010801. doi: 10.1117/1.NPh.3.1.010801

Takizawa, R., Fukuda, M., Kawasaki, S., Kasai, K., Mimura, M., Pu, S., et al. (2014). Neuroimaging-aided differential diagnosis of the depressive state. Neuroimage 85, 498-507. doi: 10.1016/j.neuroimage.2013.05.126

Thompson-Schill, S. L., D’Esposito, M., Aguirre, G. K., and Farah, M. J. (1997). Role of left inferior prefrontal cortex in retrieval of semantic knowledge: a reevaluation. Proc. Natl. Acad. Sci. U.S.A. 94, 14792-14797. doi: 10.1073/pnas.94.26.14792

Wagner, S., Sebastian, A., Lieb, K., Tüscher, O., and Tadić, A. (2014). A coordinate-based ALE functional MRI meta-analysis of brain activation during verbal fluency tasks in healthy control subjects. BMC Neurosci. 15:19. doi: 10.1186/1471-2202-15-19

Woodard, J. L., Seidenberg, M., Nielson, K. A., Antuono, P., Guidotti, L., Durgerian, S., et al. (2009). Semantic memory activation in amnestic mild cognitive impairment. Brain 132, 2068-2078. doi: 10.1093/brain/awp157

Yankner, B. A., Lu, T., and Loerch, P. (2008). The aging brain. Annu. Rev. Pathol. 3, 41-66. doi: 10.1146/annurev.pathmechdis.2.010506.092044

Yeung, M. K., Sze, S. L., Woo, J., Kwok, T., Shum, D. H., Yu, R., et al. (2016). Altered frontal lateralization underlies the category fluency deficits in older adults with mild cognitive impairment: a near-infrared spectroscopy study. Front. Aging Neurosci. 8:59. doi: 10.3389/fnagi.2016.00059

Zanto, T. P., Rubens, M. T., Thangavel, A., and Gazzaley, A. (2011). Causal role of the prefrontal cortex in top-down modulation of visual processing and working memory. Nat. Neurosci. 14, 656-661. doi: 10.1038/nn.2773

Conflict of Interest Statement: The authors declare that the research was conducted in the absence of any commercial or financial relationships that could be construed as a potential conflict of interest.

Copyright (c) 2017 Yap, Ung, Ebenezer, Nordin, Chin, Sugathan, Chan, Yip, Kiguchi and Tang. This is an open-access article distributed under the terms of the Creative Commons Attribution License (CC BY). The use, distribution or reproduction in other forums is permitted, provided the original author(s) or licensor are credited and that the original publication in this journal is cited, in accordance with accepted academic practice. No use, distribution or reproduction is permitted which does not comply with these terms. 